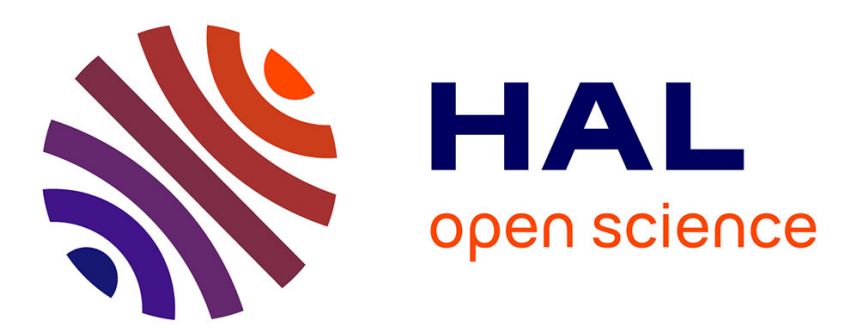

\title{
BaC4O4 - Ein kristallwasserfreies Erdalkaliquadratat mit dreidimensionaler Gerüststruktur und schichtartiger Trennung von Kationen und Anionen
}

Roberto Köferstein, Christian Robl

\section{- To cite this version:}

Roberto Köferstein, Christian Robl. BaC4O4 - Ein kristallwasserfreies Erdalkaliquadratat mit dreidimensionaler Gerüststruktur und schichtartiger Trennung von Kationen und Anionen. Journal of Inorganic and General Chemistry / Zeitschrift für anorganische und allgemeine Chemie, 2003, 629 (3), pp.371-373. 10.1002/zaac.200390061 . hal-02004576

\author{
HAL Id: hal-02004576 \\ https://hal.science/hal-02004576
}

Submitted on 1 Feb 2019

HAL is a multi-disciplinary open access archive for the deposit and dissemination of scientific research documents, whether they are published or not. The documents may come from teaching and research institutions in France or abroad, or from public or private research centers.
L'archive ouverte pluridisciplinaire HAL, est destinée au dépôt et à la diffusion de documents scientifiques de niveau recherche, publiés ou non, émanant des établissements d'enseignement et de recherche français ou étrangers, des laboratoires publics ou privés. 
$\mathrm{BaC}_{4} \mathrm{O4}_{4}$ - An Alkaline-Earth Squarate Free from Water of Crystallization with

Three-dimensional Framework Structure Featuring a Layer-like Separation of Cations and Anions

R. Köferstein, C. Robl

Z. Anorg. Allg. Chem. 629 (2003) 371-373.

DOI: $10.1002 /$ zaac.200390061

\section{Abstract}

Colourless single crystals of $\mathrm{BaC}_{4} \mathrm{O}_{4}$ have been obtained from aqueous solution at $80{ }^{\circ} \mathrm{C}$. $\mathrm{BaC}_{4} \mathrm{O}_{4}$ is stable in air up to $490{ }^{\circ} \mathrm{C}$. $\mathrm{BaCO}_{3}$ is formed by further increase of temperature. $\mathrm{BaC}_{4} \mathrm{O}_{4}$ crystallizes in the tetragonal space group $\mathrm{I} / \mathrm{mcm}$ (nr. 140) with $\mathrm{a}=635.95(5)$, $\mathrm{c}=1240.77(13) \mathrm{pm}, \mathrm{Z}=4 . \mathrm{Ba}^{2+}$ is coordinated by eight oxygen atoms of the squarate dianions; $\mathrm{Ba}-\mathrm{O}$ 276.1(1) pm. The coordination polyhedron is a distorted, square anti-prism. The squarate dianions occupy crystallographic mirror planes and posses approximately $4 / \mathrm{mmm}$ symmetry; $\mathrm{C}-\mathrm{C} 145.7(5)$ and $146.5(5) \mathrm{pm}, \mathrm{C}-\mathrm{O}$ 125.9(3) pm. A layer-like separation between the cations and anions exists with respect to the [001] direction.

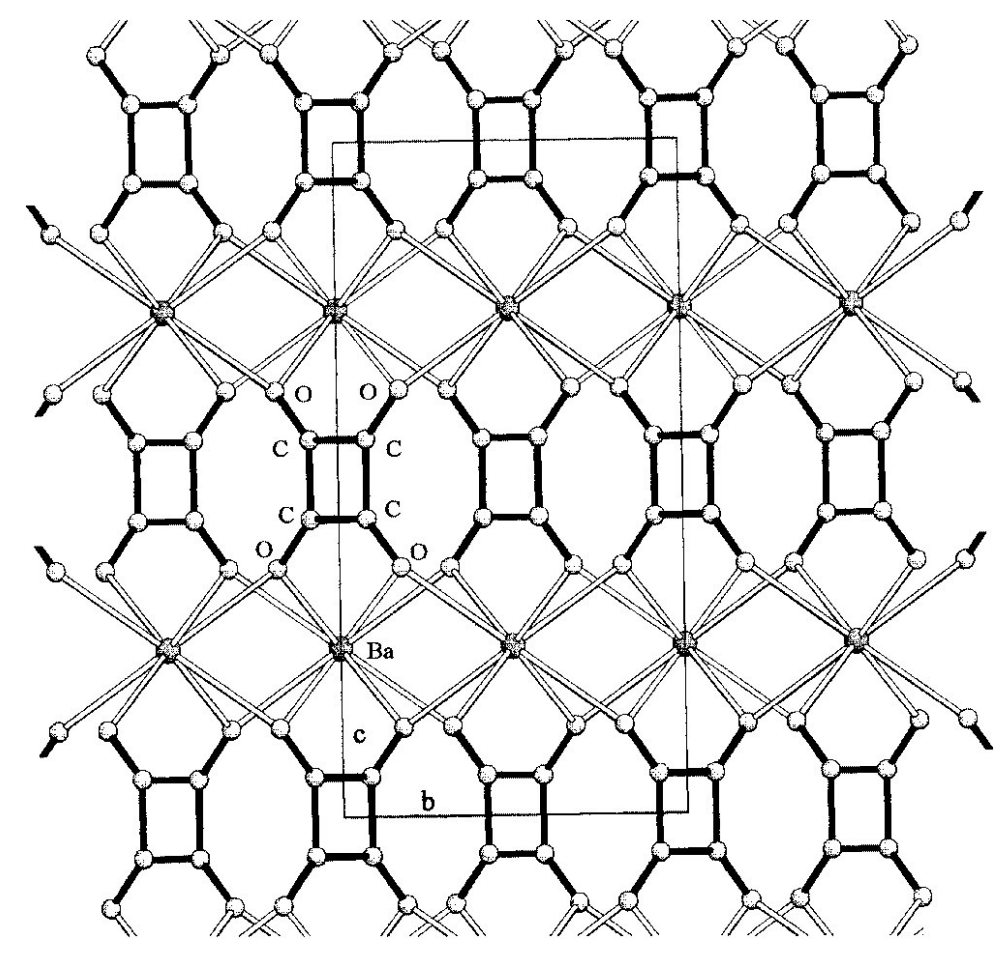




\title{
$\mathrm{BaC}_{4} \mathrm{O}_{4}$ Ein kristallwasserfreies Erdalkaliquadratat mit dreidimensionaler Gerüststruktur und schichtartiger Trennung von Kationen und Anionen
}

\author{
Roberto Köferstein und Christian Robl* \\ Jena, Institut für Anorganische und Analytische Chemie der Friedrich-Schiller-Universität
}

Professor Hartmut Bärnighausen zum 70. Geburtstag gewidmet

\section{$\mathrm{BaC}_{4} \mathrm{O}_{4}$ An Alkaline-Earth Squarate Free from Water of Crystallization with Three-dimensional Framework Structure Featuring a Layer-like Separation of Cations and Anions}

\begin{abstract}
Colourless single crystals of $\mathrm{BaC}_{4} \mathrm{O}_{4}$ have been obtained from aqueous solution at $\quad 80^{\circ} \mathrm{C} . \mathrm{BaC}_{4} \mathrm{O}_{4}$ is stable in air up to $490{ }^{\circ} \mathrm{C} . \mathrm{BaCO}_{3}$ is formed by further increase of temperature. $\mathrm{BaC}_{4} \mathrm{O}_{4}$ crystallizes in the tetragonal space group $\mathrm{I} 4 / \mathrm{mcm}$ (nr. 140) with a $=635.95(5), \mathrm{c}=1240.77(13) \mathrm{pm}, \mathrm{Z}=4 . \mathrm{Ba}^{2+}$ is coordinated by eight oxygen atoms of the squarate dianions; Ba-O 276.1(1) pm. The coordination polyhedron is a distorted, square anti-prism.
\end{abstract}

The squarate dianions occupy crystallographic mirror planes and posses approximately 4/mmm symmetry; C-C 145.7(5) and 146.5(5) pm, C-O 125.9(3) pm. A layer-like separation between the cations and anions exists with respect to the [001] direction.

Keywords: Barium squarate; Coordination polymer
Quadratsäure zählt zu den sogenannten Oxokohlenstoffsäuren mit der allgemeinen Formel $\mathrm{C}_{n} \mathrm{O}_{n} \mathrm{H}_{2}$. Das resonanzstabilisierte Quadratatdianion $\mathrm{C}_{4} \mathrm{O}_{4}{ }^{2}$ bietet sich als anionischer Baustein zum Auf-bau von Koordinationspolymeren mit verschiedenartigen Vernetzungsmustern an [1-9], wobei es sich durch besondere koordinationschemische Flexibilität auszeichnet. Ein einfacher Zusammen-hang zwischen der Summenformel des Quadratats und seiner Kristallstruktur besteht nicht. Selbst formelgleiche Verbindungen können unterschiedliche Strukturtypen haben. So sind Erdalkaliquadratate der Zusammensetzung $\mathrm{EAC}_{4} \mathrm{O}_{4} \cdot 3 \mathrm{H}_{2} \mathrm{O}$ (EA Be, Sr, Ba) bekannt, die miteinander wenig strukturelle Verwandtschaft aufweisen und durch geringe Kristallsymmetrie gekennzeichnet sind [10 15]. $\mathrm{BaC}_{4} \mathrm{O}_{4}$ konnte kristallwasserfrei aus wässeriger Lösung hergestellt werden und hat vergleichsweise hohe Kristallsymmetrie.

\section{Ergebnisse und Diskussion}

Die Kristallstruktur des $\mathrm{BaC}_{4} \mathrm{O}_{4}$ weist hohe Symmetrie auf. $\mathrm{Ba}^{2+}$ besetzt die vierzählige spezielle Punktlage 4 a der Raumgruppe I4/ $\mathrm{mcm}$ mit der Punktgruppensymmetrie 422. $\mathrm{Ba}^{2+}$ ist von acht Sauerstoffatomen aus den Quadratatdianionen in Form eines verzerrten quadratischen Antiprismas umgeben, wobei die quadratischen Basisflächen um einen Winkel von $31.7^{\circ}$ gegeneinander verdreht sind (Abb. 1). Der Ba-O-Abstand beträgt 276.1(1) pm (Tab.1),

\footnotetext{
* Prof. Dr. Christian Robl Friedrich-

Schiller-Universität Jena

Institut für Anorganische und Analytische Chemie

August-Bebel-Str. 68

D-07743 Jena

Fax: 49 (0)3641 948152 e-

mail: crr@uni-jena.de
}

woraus sich nach der Methode von Brown [16] eine Bindungsordnung von 2.21 ergibt, die etwa 10\% über dem Erwartungswert liegt. Der kürzeste $\mathrm{Ba}^{2} \mathrm{Ba}^{2+}$-Abstand mißt 449.68(4) pm und liegt damit etwa 3\% über dem Ba-Ba-Abstand in metallischem Barium (435 pm, A2-Typ).

Das Sauerstoff- und das Kohlenstoffatom des Quadratatdianions besetzen die spezielle sechzehnzählige Punktlage 161 der Raumgruppe $\mathrm{I} 4 / \mathrm{mcm}$. Es resultiert daraus ein auf einer kristallographischen Spiegelebene gelegenes und somit planares $\mathrm{C}_{4} \mathrm{O}_{4}{ }^{2-}$-Ion mit C-CBindungslängen, die sich im Rahmen der experimentellen Fehlergrenze nicht voneinander unterscheiden (145.7(5) und 146.6(5) pm, Tab. 1). Dies und die C-O-Bindungslänge von 125.9(3) pm sprechen für eine Delokalisierung des $\pi$-Elektronensystems. Die O-C C-Bindungswinkel weichen nur wenig von $135^{\circ} \mathrm{ab}(134.17(13)$ und 135.83(13) ${ }^{\circ}$, Tab. 1), so daß das $\mathrm{C}_{4} \mathrm{O}_{4}{ }^{2-}$-Ion in guter Näherung der Punktsymmetriegruppe $4 / \mathrm{mmm}$ zugeordnet werden kann. Die Sauerstoffatome des $\mathrm{C}_{4} \mathrm{O}_{4}{ }^{2}$ stehen mit jeweils drei Bindungspartnern $\left(1 \mathrm{x} \mathrm{C}, 2 \mathrm{x} \mathrm{Ba}^{2+}\right.$ ) ähnlich wie in $\mathrm{Ag}_{2} \mathrm{C}_{4} \mathrm{O}_{4}(1 \mathrm{x} \mathrm{C}, 2 \mathrm{x} \mathrm{Ag})$ [8] in Kontakt.

Die $\mathrm{Ba}^{2+}$ - und $\mathrm{C}_{4} \mathrm{O}_{4}{ }^{2-}$-Ionen bilden eine dreidimensionale Struktur mit schichtartiger Trennung der Kationen und Anionen in [001] Richtung (Abb. 1). Die mit $\mathrm{C}_{4} \mathrm{O}_{4}{ }^{2-}$-Ionen besetzten Schichten zeigen ...ABAB...-Stapelung, wobei die Ebenen der Anionen in benachbarten Schichten senkrecht zueinander orientiert sind (Abb. 2).

Die C-C- und C-O-Bindungslängen (C-O 124.4(1) 125.5(1), C-C 145.6(1) 147.7(1) pm [15]) in $\mathrm{BaC}_{4} \mathrm{O}_{4} \cdot 3 \mathrm{H}_{2} \mathrm{O}$, differieren nur wenig und entsprechen weitgehend denen in $\mathrm{BaC}_{4} \mathrm{O}_{4}$, obgleich die Koordination der Sauerstoffatome des $\mathrm{C}_{4} \mathrm{O}_{4}{ }^{2-}$-Ions im Bariumquadratat Trihydrat sehr ungleichmäßig ist. Dabei sind zwei Sauerstoffatome des Anions jeweils mit einem $\mathrm{Ba}^{2+}$, eines mit zwei $\mathrm{Ba}^{2+}$ und das vierte gar nicht mit $\mathrm{Ba}^{2+}$ verbunden (Ba-O 271.3(1) 


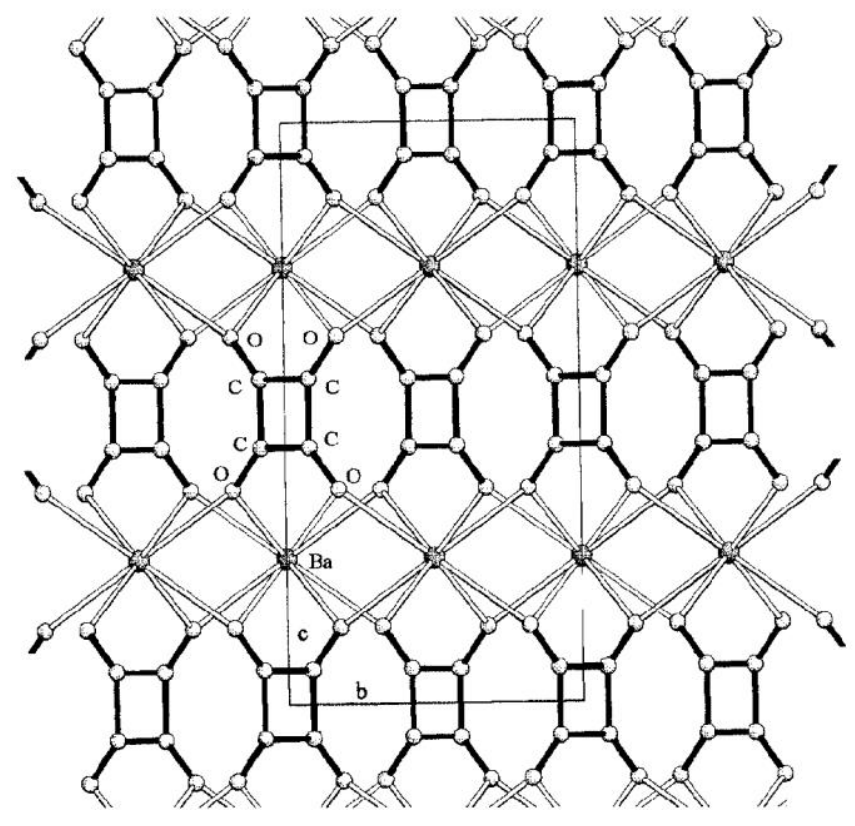

Abb. 1 Blick entlang [100]: $\mathrm{Ba}^{2+}$ wird von den Sauerstoffatomen der $\mathrm{C}_{4} \mathrm{O}_{4}{ }^{2-}$-Ionen in Form eines verzerrten quadratischen Antiprismas koordiniert. Es resultiert ein dreidimensional unendlicher Ver-

band aus $\mathrm{Ba}^{2}$-Ionen und $\mathrm{C}_{4} \mathrm{O}_{4}{ }^{2}$-Ionen. In [001] Richtung sind Kationen und Anionen schichtartig voneinander separiert.

Tabelle 1 Interatomare Abstände (in pm) und Winkel (in ${ }^{\circ}$ )

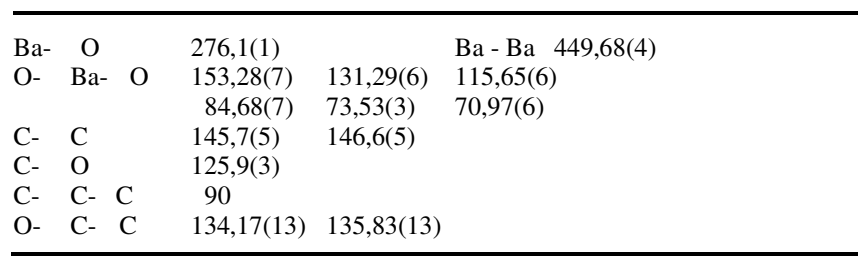

285.2(1) pm [15]). Eine Neutronenbeugungsanalyse [15] bestätigte, daß diese ungleichmäßige Umgebung durch $\mathrm{Ba}^{2+}$ im Trihydrat durch Protonen aus Wasserstoffbrückenbindungen kompensiert wird. So tritt das nicht an $\mathrm{Ba}^{2}$ gebundene Sauerstoffatom des An-ions als Protonenakzeptor in drei Wasserstoffbrückenbindungen auf. Die jeweils mit einem $\mathrm{Ba}^{2}$ koordinierten Quadratat-Sauerstoffatome sind an je einer Wasserstoffbrücke beteiligt, während das mit zwei $\mathrm{Ba}^{2+}$ in Kontakt stehende Sauerstoffatom des $\mathrm{C}_{4} \mathrm{O}_{4}{ }^{2-}$ an keiner der Wasserstoffbrïckenbindungen teilnimmt.

\section{Experimentelles}

Einkristalle von $\mathrm{BaC}_{4} \mathrm{O}_{4}$ wurden durch Kristallisation in wässeriger Lösung erhalten. Dazu wurden $10 \mathrm{ml}$ einer $0.1 \mathrm{M}$ NatriumquadratatLösung erhitzt und mit $10 \mathrm{ml}$ einer $1 \mathrm{M}$ Bariumnitrat-Lösung sowie 20 $\mathrm{ml}$ Wasser versetzt. Die Mischung wurde bei $80{ }^{\circ} \mathrm{C}$ stehen gelassen. Nach 48 h bildeten sich farblose Kristalle von $\mathrm{BaC}_{4} \mathrm{O}_{4}$.

Das IR-Spektrum von $\mathrm{BaC}_{4} \mathrm{O}_{4}$ zeigt die für das Quadratatdianion typischen Absorptionsbanden. Eine scharfe Bande bei $1088 \mathrm{~cm}^{-1} \mathrm{kann}$ der C-C-Streckschwingung zugeordnet werden. Die C-OStreckschwingung verursacht eine Absorption bei $1451 \mathrm{~cm}^{-1}$. Nach der Methode von Siebert [17] errechnet sich hieraus die

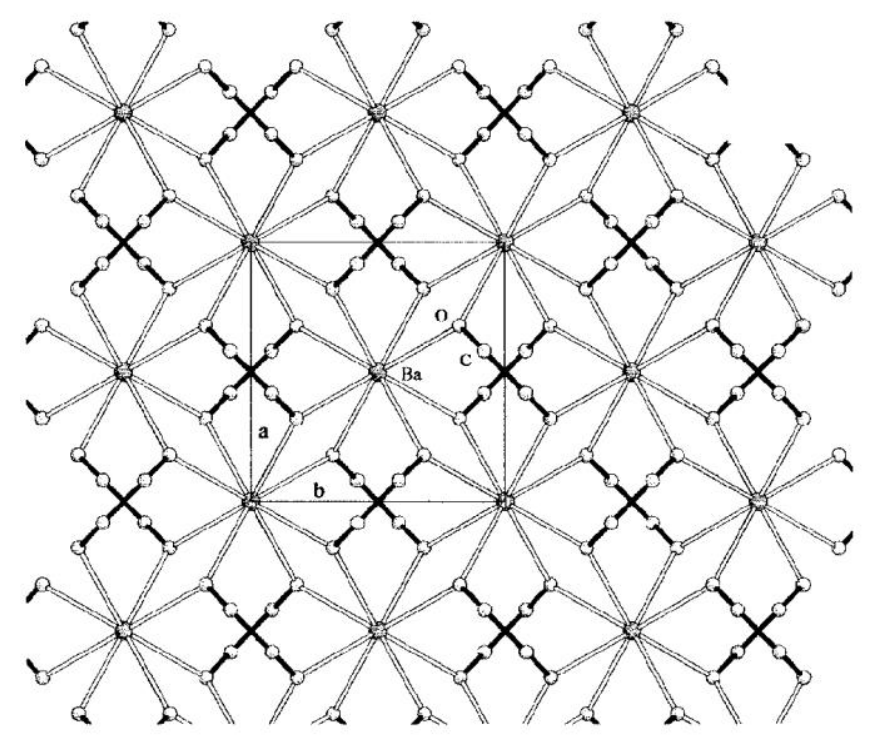

Abb. 2 Blick auf (001): Die $\mathrm{C}_{4} \mathrm{O}_{4}{ }^{2-}$-Ionen sind entlang kristallographischer Spiegelebenen ausgerichtet. Die aus Anionen gebildeten Schichten sind in ...ABAB... Folge in [001] Richtung gestapelt. Die Ebenen von Anionen benachbarter Schichten stehen senkrecht zueinander.

Tabelle 2 Kristallographische Daten für $\mathrm{BaC}_{4} \mathrm{O}_{4}$

\begin{tabular}{|c|c|}
\hline Summenformel & $\mathrm{BaC}_{4} \mathrm{O}_{4}$ \\
\hline Kristallsystem & tetragonal \\
\hline Raumgruppe & $\mathrm{I} 4 / \mathrm{mcm}$ (Nr. 140$)$ \\
\hline Gitterkonstanten & $\begin{array}{l}\mathrm{a}=635.95(5) \mathrm{pm} \\
\mathrm{c}=1240.77(13) \mathrm{pm}\end{array}$ \\
\hline Elementarzellvolumen & $0.50181(8) \mathrm{nm}^{3}$ \\
\hline Formeleinheiten in der EZ & $Z=4$ \\
\hline Molmasse & $249.38 \mathrm{~g} / \mathrm{mol}$ \\
\hline berechnete Dichte & $3.301 \mathrm{Mg} / \mathrm{m}^{3}$ \\
\hline Diffraktometer & Siemens P4 \\
\hline Strahlung & $\mathrm{MoK} \alpha$ \\
\hline Monochromator & Graphit \\
\hline Absorptionskoeffizient & $7.832 \mathrm{~mm}^{-1}$ \\
\hline Absorptionskorrektur & numerisch \\
\hline min./max. Transmission & $0.53 / 0.57$ \\
\hline Meßtemperatur & $293 \mathrm{~K}$ \\
\hline Meßmodus & $\omega$-scan \\
\hline Kristallabmessungen & $0.08 \mathrm{~mm} \times 0.08 \mathrm{~mm} \times 0.20 \mathrm{~mm}$ \\
\hline$\Theta$-Bereich & $3.2526 .97^{\circ}$ \\
\hline$F(000)$ & 448 \\
\hline Oktanten & Vollkugel \\
\hline gemessene Reflexe & 1962 \\
\hline davon symmetrieunabhängig & 163 \\
\hline $\mathrm{K}_{\text {int }}$ & 0.0235 \\
\hline Strukturlösung & Direkte Methoden \\
\hline Strukturverfeinerung & Vollmatrix-LS mit $\mathrm{F}^{2}$ \\
\hline Extinktionskoeffizient & $0.0055(6)$ \\
\hline Residualwerte (alle Daten) & $\mathrm{R}_{1} 0.0112$ \\
\hline & $w \mathrm{R}_{2} \quad 0.0258$ \\
\hline Extrema der letzten Differenz-Fourier-Synthese & $515 / \quad 363 \mathrm{e} / \mathrm{nm}^{3}$ \\
\hline Programm & SHELXTL [18] \\
\hline
\end{tabular}

Bindungsordnung der C-O-Bindung zu 1,4. Dies ist in guter Übereinstimmung mit den röntgenstrukturanalytischen Ergebnissen, die wie bei anderen Metallquadrataten eine Delokalisierung des $\pi$ Elektronensystems ausweisen.

$\mathrm{BaC}_{4} \mathrm{O}_{4}$ wurde thermoanalytisch in einer Netzsch STA 429-Ap-paratur an Luft im Temperaturbereich von $25800{ }^{\circ} \mathrm{C}$ untersucht. Die Verbindung erwies sich bis zu einer Temperatur von etwa $490{ }^{\circ} \mathrm{C}$ als stabil. Bei weiterer Temperaturerhöhung erfolgte ein exothermer Vorgang, der bei $620^{\circ} \mathrm{C}$ abgeschlossen und mit einem 
Tabelle 3 Atomkoordinaten und Koeffizienten $\left(\mathrm{pm}^{2} \times 10^{-1}\right)$ des anisotropen Temperaturfaktors für $\mathrm{BaC}_{4} \mathrm{O}_{4}$

\begin{tabular}{|c|c|c|c|c|c|c|c|c|c|c|}
\hline Atom & $\mathrm{x}$ & $\mathrm{y}$ & $\mathrm{z}$ & $\mathrm{U}_{11}$ & $\mathrm{U}_{22}$ & $\mathrm{U}_{33}$ & $\mathrm{U}_{23}$ & $\mathrm{U}_{13}$ & $\mathrm{U}_{12}$ & $\mathrm{U}_{\mathrm{eq}}$ \\
\hline $\mathrm{Ba}$ & 0 & 0 & 0.25 & 11(1) & 11(1) & $11(1)$ & 0 & 0 & 0 & 11(1) \\
\hline $\mathrm{O}$ & $0.1791(2)$ & $0.3209(2)$ & $0.1315(1)$ & $16(1)$ & $16(1)$ & $14(1)$ & $4(1)$ & $4(1)$ & $0(1)$ & $16(1)$ \\
\hline $\mathrm{C}$ & $0.0815(3)$ & $0.4185(3)$ & $0.0587(2)$ & $10(1)$ & $10(1)$ & $13(1)$ & $0(1)$ & $0(1)$ & $3(1)$ & 11(1) \\
\hline
\end{tabular}

Gewichtsverlust von $16 \%$ verbunden war. Im festen Rückstand konnte $\mathrm{BaCO}_{3}$ (Witherit) röntgenographisch als Reaktionsprodukt nachgewiesen werden.

In Tabelle 2 sind die Angaben zur Kristallstrukturbestimmung zusammengefaßt. Die Atomparameter können der Tabelle 3 entnommen werden. Weitere Einzelheiten zur Kristallstrukturbestimmung sind beim Fachinformationszentrum Karlsruhe, D-76344 EggensteinLeopoldshafen, unter Angabe der Hinterlegungsnummer CSD 412830, der Autorennamen und des Zeitschriftenzitats erhältlich.

Wir danken dem Fonds der Chemischen Industrie für finanzielle Unterstützung.

[1] R. West, H. Y. Niu, J. Am. Chem. Soc. 1963, 85, 2589.

[2] J. A. C. van Ooijen, I. Reedijk, A. C. Spek, Inorg. Chem. 1979, $18,1184$.

[3] A. Weiss, E. Riegler, C. Robl, Z. Naturforsch. 1986, 41b, 1329.
[4] A. Weiss, E. Riegler, C. Robl, Z. Naturforsch. 1986, 41b, 1333.

[5] C. Robl, W. F. Kuhs, J. Solid State Chem. 1988, 75, 15.

[6] C. Robl, A. Weiss, Z. Naturforsch. 1986, 41b, 1341.

[7] C. Robl, A. Weiss, Mat. Res. Bull. 1987, 22, 373.

[8] C. Robl, A. Weiss, Z. Anorg. Allg. Chem. 1987, 546, 161.

[9] J.-C. Trombe, J.-C. Petit, A. Gleizes, New J. Chem. 1988, 12 , 197.

[10] C. Robl, D. Kinkeldey, Z. Naturforsch. 1990, 45b, 931.

[11] C. Robl, A. Weiss, Z. Naturforsch. 1986, 41b, 1490.

[12] C. Robl, V. Gnutzmann, A. Weiss, Z. Anorg. Allg. Chem. 1987, $549,187$.

[13] C. Robl, Z. Naturforsch. 1988, 43b, 99.

[14] C. Robl, A. Weiss, Z. Naturforsch. 1986, 41b, 1485.

[15] C. Robl, W. F. Kuhs, J. Solid State Chem. 1988, 73, 172.

[16] I. D. Brown, K. K. Wu, Acta Crystallogr. 1976, B24, 63.

[17] H. Siebert, Z. Anorg. Allg. Chem. 1953, 273, 170.

[18] G. M. Sheldrick, SHELXTL, Bruker AXS Inc., Madison, Wisconsin, USA, 1994. 